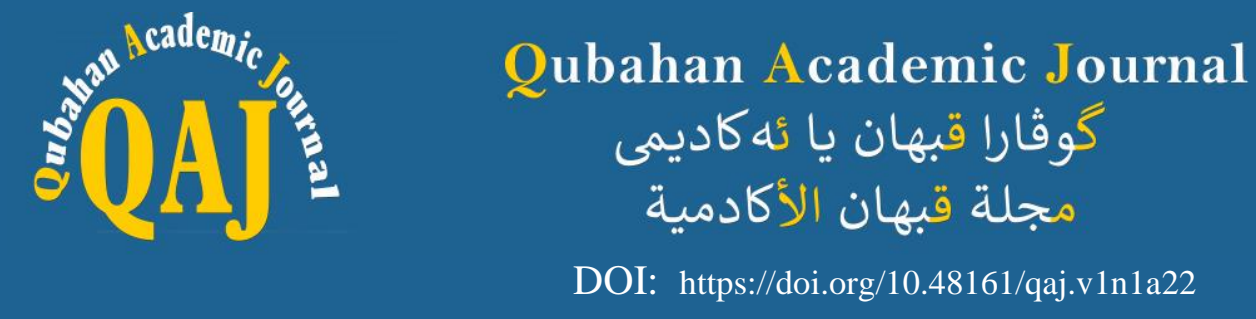

\title{
Variation Comparison of OLS and GLS Estimators using Monte Carlo Simulation of Linear Regression Model with Autoregressive Scheme
}

\author{
Sajid Ali Khan \\ Green Hills Postgraduate College \\ Rawalakot Poonch AJK \\ Rawalakot, Pakistan \\ sajid.al680@gmail.com
}

\author{
Sayyad Khurshid \\ Government Postgraduate College \\ Boys Rawalakot AJK \\ Rawalakot, Pakistan \\ sayyadkhurshid8@gmail.com \\ Kashmala Khurshid \\ Government Postgraduate College \\ Boys Rawalakot AJK \\ Rawalakot, Pakistan \\ kashmalakhurshid44@gmail.com
}

\author{
Tooba Akhtar \\ Government Postgraduate College \\ Boys Rawalakot AJK \\ Rawalakot, Pakistan \\ toobasohail889@gmail.com
}

\begin{abstract}
In this research we discuss to Ordinary Least Squares and Generalized Least Squares techniques and estimate with First Order Autoregressive scheme from different correlation levels by using simple linear regression model. A comparison has been made between these two methods on the basis of variances results. For the purpose of comparison, we use simulation of Monte Carlo study and the experiment is repeated 5000 times. We use sample sizes 50, 100, 200,300 and 500, and observe the influence of different sample sizes on the estimators.
\end{abstract}

By comparing variances of OLS and GLS at different values of sample sizes and correlation levels with $\sigma=1,3$, we found that variance of $\left(\begin{array}{l}\beta \\ 0\end{array}\right)$ at sample size 500, OLS and GLS gives similar results but at sample size 50 variance of GLS $(\beta$ 0) has minimum values as compared to OLS. So, it is clear that variance of GLS $\left(\beta \_0\right)$ is best. Similarly, variance of $\left(\beta \_1\right)$ from OLS and GLS at sample size 500 and correlation $-\mathbf{0 . 0 5}$ with $\sigma=1$, GLS give minimum value as compared to all other sample sizes and correlations.

By comparing overall results of Ordinary Least Squares (OLS) and Generalized Least Squares (GLS), we conclude that in large samples both are gives similar results but small samples GLS is best fitted as compared to OLS.

Keywords- Linear Regression, Comparison, OLS, GLS, Autoregressive Scheme

\section{INTRODUCTION}

In regression modeling, first-order auto correlated errors are often a problem. When the data also suffers from independent variables, generalized least squares estimation is no longer the best alternative to ordinary least squares. The Monte Carlo simulation illustrates that regression estimation using data transformed according to the generalized least squares method provides estimates of the regression coefficients which are superior to generalized least squares and ordinary least squares estimates.

While trying to beat the shortcomings of conventional least squares assessment strategy within the sight of autocorrelation, this investigation looks to apply the summed up least squares assessment technique since the least squares assessment technique doesn't utilize the data of the unexplained change as caught by the mistake terms in the needy variable, though the Generalized Least Squares (GLS) takes such data, the unexplained difference into account unequivocally and is refined.

First order autocorrelation refers to a one period lag relationship between the consecutive errors in a time series regression model. This violation of assumption that the errors are uncorrelated an in this situation, the best linear unbiased estimator (BLUE) of the regression equation is the generalized least squares (GLS) estimator. Auto correlated errors occur most frequently in econometrics and time series modeling because of the trend present in the cumulative effects of the omitted variables in the regression setting. The regression equations are often used for prediction and forecasting (Virgantari, Wijayanti, \& Koeshendrajana, 2018).

Monte Carlo simulation depends on the cycle of unequivocally speaking to vulnerabilities by indicating contributions as likelihood conveyances. On the off chance that the data sources portraying a framework are questionable, the forecast of future execution is essentially dubious. Monte Carlo reproduction methods have been utilized to create information undefined from information gathered from genuine marvels that clung to the details of 
our model. Monte Carlo methods are along these lines exceptionally fit to experimentally contemplating the properties of hypothetical models and this is the means by which they're regularly utilized (Kassab \& Awjar, 2020).

\section{OBJECTIVE OF THE STUDY}

The main objectives are:

- To estimate the parameters using Monte Carlo Simulations with different samples.

- To compare the variances OLS and GLS estimators in linear regression model with First Order AutoRegressive scheme.

\section{LITERATURE REVIEW}

Safi \& Saif (2014) dissected a significant measurable issue concerning determining in relapse models in time arrangement measures. It is notable that the most acclaimed strategy for assessing and anticipating is the Ordinary Least Squares (OLS). OLS might be not the ideal in this specific circumstance. So throughout the long term many specific assessment strategies have been created, for instance Generalized Least Squares (GLS). We are contrasting the anticipating dependent on certain assessors with the forecast utilizing the GLS gauge. This correlation will be utilized by what is known as proportions of figure exactness. We lead a broad PC reproduction time arrangement information, to make correlation among these techniques.

Akpan \& Moffat (2018) dissected the summed up least squares to conquer the shortcomings of common least squares to guarantee the productivity of the model boundaries, fair standard mistakes, substantial $t$ insights and p-values, and to represent the presence of autocorrelation. Notwithstanding, the conclusion of the fitted relapse model utilizing Breusch and Godfrey test, ACF, and PACF indicated that the residuals are related, henceforth the requirement for summed up least squares. Further discoveries from the aftereffects of summed up least squares assessment uncovered that their appraisals are better and that the extra data in the blunder terms (autocorrelation) could be clarified and caught by AR (2). Subsequently, it very well may be derived that summed up least squares gives preferred appraisals over the normal least settles and furthermore outstanding balances for autocorrelation in time arrangement relapse examination.

Virgantari, Wijayanti, \& Koeshendrajana (2018) talked about Ordinary Least Squares (OLS) strategy is the most well known and generally method used to assess mathematical estimations of boundaries of chose relapse models. This was because of its fair-mindedness property. Nonetheless, when components of ward variable have inconsistent differences and additionally related one another, there is no assurance that the OLS assessor will show the most proficient inside the class of straight fair-minded assessors. For conditions commonly experienced, GLS technique is proposed an assessment system which yields coefficient assessors in any event asymptotically more proficient than single condition OLS assessor. This technique is inferred by (Aitken, 1935) and it's named Aitken GLS. This exploration revealed an investigation of utilization of Aitken GLS strategy for assessing boundary of interest capacity of creature protein in Indonesia of which has a framework condition. This arrangement of condition causes infringement of the presumptions of homoscedasticity and independency of assessed boundaries.

Wenning \& Valenci (2019) talked about the decency of fit for an ARMA time arrangement model that a portmanteau trial of the residuals is led to evaluate remaining sequential relationship of the fitted ARMA model. Of the numerous portmanteau tests accessible for this reason, one of the most celebrated and generally utilized is a variation of the first Box-Pierce test, the Ljung-Box test. Notwithstanding the fame of this test, in any case, there are a few other more current portmanteau tests accessible to evaluate lingering sequential autocorrelation of the fitted ARMA model. This examination centers around the aftereffects of a force investigation contrasting these three distinct tests against various attacks of ARMA inferred time arrangement, just as the conduct of the three diverse test insights inspected when applied to a genuine informational index. We affirm that for circumstances where the moving normal part of a fitted ARMA model is disparaged or when the example size is little.

Chang \& Peloquin (2020) proposed an improved calculation. The new calculation includes just two lattice factorizations, rather than three, and can be executed by hindering procedures. It show that, regarding flop checks, the improved calculation costs not as much as Paige's first calculation regardless and not as much as his second calculation at times. Mathematical tests show that regarding reproductions running time, our improved calculation is quicker than both of the current calculations when hindering procedures are utilized.

Kassab \& Awjar (2020) analyzed correlation between the explanatory variables in multiple linear regression raises the variances of the least squares estimators. These estimators become unstable and may have wrong signs. A Monte Carlo method is used to estimate these regression parameters and a comparison between the ordinary least squares and the new ridge regression parameter methods was made in the sense of having smaller mean squares error. Based on simulation study, it found that the new ridge regression method where the ridge parameter $\mathrm{k}$ is a vector performs better than the ordinary least squares method.

Kibria \& Lukman (2020) acquainted another one-sided assessor with defeat the multicollinearity issue for the various straight relapse models and gave the assessment method of the biasing boundary. A reproduction study has been led to think about the exhibition of the proposed assessor and Liu (1993) and edge relapse assessors. Reproduction results clearly show that the proposed assessor performed in a way that is better than both Liu and edge under some condition on the shrinkage boundary. Two arrangements of genuine information are examined to represent the advantages of utilizing the new assessor with regards to a direct relapse model.

Moon \& Gunther (2020) assessed the boundaries of the Auto-Regressive (AR) irregular cycle are a difficult that has been very much contemplated. In numerous applications, just uproarious estimations of AR measure are accessible. The impact of the added substance commotion is that the framework can be displayed as an AR model with shaded clamor, in any event, when the estimation clamor is white, where the connection lattice relies upon the AR boundaries. 
Due to the connection, it is practical to register utilizing various stacked perceptions. Playing out a weighted leastsquares assessment of the AR boundaries utilizing a reverse covariance weighting can give essentially better boundary gauges, with progress expanding with the stack profundity. Various methods of assessing the obscure covariance are introduced, just as a technique to appraise the changes of the $\mathrm{AR}$ and perception commotion. The documentation is stretched out to Vector Auto-Regressive (VAR) measures. Recreation results exhibit execution upgrades in coefficient mistake and in range assessment.

\section{MATERIAL AND METHODS}

\section{A. Monte Carlo Simulation}

The conduct of a framework as it advances over the long run is concentrated by building up a reproduction model. This model normally appears as a series of expectations concerning the activity of the framework. Recreation can likewise be utilized to contemplate frameworks in the plan stage, before such frameworks are fabricated (Wenning \& Valenci, 2019). Accordingly, reenactment demonstrating can be utilized both as an examination instrument for foreseeing the impact of changes to existing frameworks and as a plan apparatus to anticipate the exhibition of new frameworks under shifting situations.

This simulation study is performed for different samples. For data generation and analysis, $\mathrm{R}$ programming and Minitab have been used.

\section{B. Ordinary Least Squares}

In this passage, OLS regression will be examined with regards to a bivariate model, that is, a model wherein there is just a single autonomous variable (X) anticipating a needy variable (Y). Not with standing, the rationale of OLS relapse is effortlessly stretched out to the multivariate model where there are at least two free factors (Safi \& White, 2006). The simple linear regression model is:

$$
Y=\beta_{0}+\beta_{1} X+e
$$

By definition variance

$$
\begin{aligned}
& \operatorname{Var}\left(\hat{\beta}_{\text {OLS }}\right)=E[\hat{\beta}-E(\hat{\beta})][\hat{\beta}-E(\hat{\beta})]^{t} \\
& \operatorname{Var}\left(\hat{\beta}_{\text {OLS }}\right)=E[\hat{\beta}-\beta][\hat{\beta}-\beta]^{t} \\
& \text { Where } \\
& \hat{\beta}=\left(X^{t} X\right)^{-1} X^{t} Y \\
& \therefore Y=X \beta+e \\
& \hat{\beta}=\left(X^{t} X\right)^{-1} X^{t}(X \beta+e) \\
& \hat{\beta}=\left(X^{t} X\right)^{-1} X^{t} X \beta+\left(X^{t} X\right)^{-1} X^{t} e \\
& \hat{\beta}=\beta+\left(X^{t} X\right)^{-1} X^{t} e \\
& \hat{\beta}=\beta+\left(X^{t} X\right)^{-1} X^{t} e \\
& \hat{\beta}-\beta=\left(X^{t} X\right)^{-1} X^{t} e \\
& \operatorname{Var}\left(\hat{\beta}_{\text {OLS }}\right)=E\left[\left(X^{t} X\right)^{-1} X^{t} e\right]\left[\left(X^{t} X\right)^{-1} X^{t} e\right]^{t} \\
& \operatorname{Var}\left(\hat{\beta}_{\text {OLS }}\right)=E\left[\left(X^{t} X\right)^{-1} X^{t} e\right]\left[\left(X^{t} X\right)^{-1} X e^{t}\right] \\
& \operatorname{Var}\left(\hat{\beta}_{O L S}\right)=E\left[\left(X^{t} X\right)^{-1} X^{t} e e^{t} X\left(X^{t} X\right)^{-1}\right] \\
& \operatorname{Var}\left(\hat{\beta}_{\text {oLS }}\right)=E\left[\left(X^{t} X\right)^{-1}\left(X^{t} X\right)\left(e e^{t}\right)\left(X^{t} X\right)^{-1}\right] \\
& \operatorname{Var}\left(\hat{\beta}_{\text {oLs }}\right)=E\left[e e^{t}\right]\left(X^{t} X\right)^{-1} \\
& \operatorname{Var}\left(\hat{\beta}_{\text {oLs }}\right)=\sigma_{u}^{2} I_{n}\left(X^{t} X\right)^{-1}
\end{aligned}
$$

\section{Generalized Least Squares}

The summed up Generalized Least Squares (GLS) assessor of the coefficients of a straight relapse is a speculation of the common Ordinary Least Squares (OLS) assessor. It is utilized to manage circumstances in which the OLS assessor isn't BLUE, since one of the fundamental suspicions of the Gauss-Markov hypothesis, specifically that of homoscedasticity and nonappearance of sequential relationship, is disregarded. In such circumstances, given that different suppositions of the Gauss-Markov hypothesis are fulfilled, the GLS assessor is BLUE Aitken (1935). The simple linear regression model is:

$$
Y=\beta_{0}+\beta_{1} X+e
$$

By definition variance

$$
\begin{aligned}
& \operatorname{Var}\left(\hat{\beta}_{G L S}\right)=E[\hat{\beta}-E(\hat{\beta})][\hat{\beta}-E(\hat{\beta})]^{t} \\
& \operatorname{Var}\left(\hat{\beta}_{G L S}\right)=E[\hat{\beta}-\beta][\hat{\beta}-\beta]^{t}
\end{aligned}
$$

Where

$$
\begin{aligned}
& \hat{\beta}_{G L S}=\left(X^{t} \Omega^{-1} X\right)^{-1} X^{t} \Omega^{-1} Y \\
& \therefore Y=X \beta+e \\
& \hat{\beta}_{G L S}=\left(X^{t} \Omega^{-1} X\right)^{-1} X^{t} \Omega^{-1}(X \beta+e)
\end{aligned}
$$

$$
\begin{gathered}
\hat{\beta}_{G L S}=\left(X^{t} \Omega^{-1} X\right)^{-1} X^{t} \Omega^{-1} X \beta+\left(X^{t} \Omega^{-1} X\right)^{-1} X^{t} \Omega^{-1} e \\
\hat{\beta}_{G L S}=\beta+\left(X^{t} \Omega^{-1} X\right)^{-1} X^{t} \Omega^{-1} e \\
\hat{\beta}_{G L S}-\beta=\left(X^{t} \Omega^{-1} X\right)^{-1} X^{t} \Omega^{-1} e
\end{gathered}
$$

$\operatorname{Var}\left(\hat{\beta}_{G L S}\right)=$

$E\left[\left(X^{t} \Omega^{-1} X\right)^{-1} X^{t} \Omega^{-1} e\right]\left[\left(X^{t} \Omega^{-1} X\right)^{-1} X^{t} \Omega^{-1} e\right]^{t}$

$$
\begin{aligned}
& \operatorname{Var}\left(\hat{\beta}_{G L S}\right)= \\
& E\left[\left(X^{t} \Omega^{-1} X\right)^{-1} X^{t} \Omega^{-1} e\right]\left[\left(X^{t} \Omega^{-1} X\right)^{-1} X \Omega^{-1} e^{t}\right]
\end{aligned}
$$

$$
\begin{aligned}
& \operatorname{Var}\left(\hat{\beta}_{G L S}\right)=E\left[\left(X^{t} \Omega^{-1} X\right)^{-1} X^{t} \Omega^{-1} e e^{t} \Omega^{-1} X\left(X^{t} \Omega^{-1} X\right)^{-1}\right] \\
& \operatorname{Var}\left(\hat{\beta}_{G L S}\right)= \\
& E\left[\left(X^{t} \Omega^{-1} X\right)^{-1}\left(X^{t} \Omega^{-1} X\right)\left(e e^{t}\right) \Omega^{-1}\left(X^{t} \Omega^{-1} X\right)^{-1}\right]
\end{aligned}
$$

$$
\begin{aligned}
& \operatorname{Var}\left(\hat{\beta}_{G L S}\right)= \\
& \left(X^{t} \Omega^{-1} X\right)^{-1}\left(X^{t} \Omega^{-1} X\right) E\left(e e^{t}\right) \Omega^{-1}\left(X^{t} \Omega^{-1} X\right)^{-1}
\end{aligned}
$$

$$
\begin{aligned}
& \operatorname{Var}\left(\hat{\beta}_{G L S}\right)= \\
& \left(X^{t} \Omega^{-1} X\right)^{-1}\left(X^{t} \Omega^{-1} X\right) \sigma^{2} \Omega \Omega^{-1}\left(X^{t} \Omega^{-1} X\right)^{-1}
\end{aligned}
$$

$$
\begin{aligned}
& \operatorname{Var}\left(\hat{\beta}_{G L S}\right)= \\
& \sigma^{2}\left(X^{t} \Omega^{-1} X\right)^{-1}\left(X^{t} \Omega^{-1} X\right) \Omega \Omega^{-1}\left(X^{t} \Omega^{-1} X\right)^{-1} \\
& \quad \operatorname{Var}\left(\hat{\beta}_{G L S}\right)=\sigma^{2}\left(X^{t} \Omega^{-1} X\right)^{-1}
\end{aligned}
$$




\section{First Order Auto-Regressive (AR) Scheme}

Model:

$$
e_{t}=\rho e_{t-1}+u_{t}
$$

The above equation of $e_{-} t$ is known as Markov first order autoregressive scheme, usually denoted by AR (1). The coefficient $\rho$ is called the first order autocorrelation coefficient (also called the coefficient of auto covariance) and takes values from -1 to +1 . The size of $\rho$ determines strength of autocorrelation or serial correlation (Moon \& Gunther, 2020). That is;

$$
\begin{gathered}
\hat{\rho}=\frac{\sum_{t=2}^{n} e_{t} e_{t-1}}{\sum_{t=2}^{n} e^{2} t} \\
\text { where } E\left(e e^{t}\right)=\frac{\sigma_{u}^{2}}{1-\rho^{2}}\left[\begin{array}{cccc}
1 & \rho & \ldots & \rho^{n-1} \\
\rho & 1 & \cdots & \rho^{n-2} \\
\vdots & \vdots & \vdots & \vdots \\
\rho^{n-1} & \rho^{n-2} & \ldots & 1
\end{array}\right], \text { and } \\
\sigma_{u}^{2}=\frac{\sum_{t=1}^{n} \hat{u}_{t}^{2}}{n-k}
\end{gathered}
$$

\section{RESULT AND DISCUSSION}

\section{A. Monte Carlo Simulation Algorithm}

We used Monte Carlo proposed algorithm to conduct two different simulation studies in OLS and GLS estimators in linear regression model when the errors are correlated with first order autoregressive scheme. In each step, we provide $\mathrm{R}$ programming that achieve the goals. Our algorithm has four main steps as follows:

1) Model Building

- Specify the values of model parameters

- Sample size selection

- Generation of random error values

- Generate the values of independent and dependent variables

2) Estimation of Parameters

3) Replications

Average values called Monte Carlo Estimates

\begin{tabular}{|c|c|c|c|c|c|c|c|c|c|c|}
\hline & \multicolumn{2}{|c|}{$\mathbf{n}=\mathbf{5 0}$} & \multicolumn{2}{|c|}{$\mathrm{n}=100$} & \multicolumn{2}{|c|}{$\mathbf{n}=\mathbf{2 0 0}$} & \multicolumn{2}{|c|}{$\mathrm{n}=\mathbf{3 0 0}$} & \multicolumn{2}{|c|}{$\mathrm{n}=\mathbf{5 0 0}$} \\
\hline & $\beta_{0}$ & $\beta_{1}$ & $\beta_{0}$ & $\beta_{1}$ & $\beta_{0}$ & $\beta_{1}$ & $\beta_{0}$ & $\beta_{1}$ & $\beta_{0}$ & $\beta_{1}$ \\
\hline & \multicolumn{10}{|c|}{ rho $=-0.9$, var $=1$} \\
\hline $\begin{array}{l}\text { Var } \\
\text { OLS }\end{array}$ & 0.1169 & 0.3604 & 0.0539 & 0.1704 & 0.0246 & 0.0710 & 0.0174 & 0.0497 & 0.0105 & 0.0316 \\
\hline $\begin{array}{l}\text { Var } \\
\text { GLS } \\
\end{array}$ & 0.1168 & 0.3603 & 0.0539 & 0.1704 & 0.0246 & 0.0710 & 0.0174 & 0.0497 & 0.0105 & 0.0316 \\
\hline & \multicolumn{10}{|c|}{ rho $=-0.5$, var $=1$} \\
\hline $\begin{array}{l}\text { Var } \\
\text { OLS }\end{array}$ & 0.0283 & 0.0785 & 0.0137 & 0.0438 & 0.0063 & 0.0186 & 0.0044 & 0.0133 & 0.0022 & 0.0064 \\
\hline $\begin{array}{c}\text { Var } \\
\text { GLS } \\
\end{array}$ & 0.0283 & 0.0785 & 0.0137 & 0.0438 & 0.0063 & 0.0186 & 0.0044 & 0.0133 & 0.0022 & 0.0064 \\
\hline & \multicolumn{10}{|c|}{ rho $=0.5$, var $=1$} \\
\hline $\begin{array}{l}\text { Var } \\
\text { OLS }\end{array}$ & 0.2493 & 0.2438 & 0.1118 & 0.1070 & 0.0521 & 0.0466 & 0.0328 & 0.0264 & 0.0187 & 0.0171 \\
\hline $\begin{array}{c}\text { Var } \\
\text { GLS } \\
\end{array}$ & 0.2080 & 0.1197 & 0.1108 & 0.0605 & 0.0519 & 0.0265 & 0.0328 & 0.0168 & 0.0186 & 0.0099 \\
\hline & \multicolumn{10}{|c|}{ rho $=0.9$, var $=1$} \\
\hline $\begin{array}{l}\text { Var } \\
\text { OLS }\end{array}$ & 112.328 & 5.2785 & 26.4752 & 3.2579 & 9.4950 & 1.5402 & 5.5008 & 0.8131 & 6.2541 & 0.5811 \\
\hline $\begin{array}{c}\text { Var } \\
\text { GLS }\end{array}$ & 105.420 & 0.5324 & 24.1893 & 0.3490 & 9.0764 & 0.1366 & 5.3486 & 0.0853 & 6.1088 & 0.0587 \\
\hline & \multicolumn{10}{|c|}{$\mathrm{rho}=-0.9, \mathrm{var}=3$} \\
\hline $\begin{array}{l}\text { Var } \\
\text { OLS }\end{array}$ & 0.3293 & 1.1039 & 0.1589 & 0.4966 & 0.0717 & 0.1998 & 0.0516 & 0.1533 & 0.0295 & 0.0839 \\
\hline $\begin{array}{c}\text { Var } \\
\text { GLS } \\
\end{array}$ & 0.3293 & 1.1038 & 0.1589 & 0.4965 & 0.0717 & 0.1997 & 0.0516 & 0.1533 & 0.0295 & 0.8394 \\
\hline & \multicolumn{10}{|c|}{ rho $=-0.5$, var $=3$} \\
\hline $\begin{array}{l}\text { Var } \\
\text { OLS }\end{array}$ & 0.0841 & 0.2532 & 0.0412 & 0.1140 & 0.0191 & 0.0509 & 0.0127 & 0.0383 & 0.0063 & 0.0198 \\
\hline $\begin{array}{c}\text { Var } \\
\text { GLS } \\
\end{array}$ & 0.0841 & 0.2532 & 0.0412 & 0.1140 & 0.0191 & 0.0509 & 0.0127 & 0.0383 & 0.0063 & 0.0198 \\
\hline & \multicolumn{10}{|c|}{ rho $=0.5, \mathrm{rar}=3$} \\
\hline $\begin{array}{l}\text { Var } \\
\text { OLS }\end{array}$ & 0.7669 & 0.4782 & 0.3286 & 0.2786 & 0.1479 & 0.1668 & 0.0970 & 0.0902 & 0.0601 & 0.0572 \\
\hline $\begin{array}{c}\text { Var } \\
\text { GLS } \\
\end{array}$ & 0.7513 & 0.2837 & 0.3249 & 0.1572 & 0.1469 & 0.0921 & 0.0968 & 0.0547 & 0.0600 & 0.0320 \\
\hline & \multicolumn{10}{|c|}{ rho $=0.9$, var $=3$} \\
\hline Var & 282.63 & 19.206 & 92.055 & 9.9035 & 35.599 & 3.9800 & 17.539 & 2.3885 & 13.1078 & 1.6248 \\
\hline
\end{tabular}

4) Evaluation and Presentation of Results

The Monte Carlo Simulation results are given in Table 1.

TABLE I. MONTE CARlo Simulation Estimation VARying SAMPLE Sizes and CoRrelations 


\begin{tabular}{|c|c|c|c|c|c|c|c|c|c|c|}
\hline OLS & & & & & & & & & & \\
\hline $\begin{array}{c}\text { Var } \\
\text { GLS }\end{array}$ & 261.49 & 2.1179 & 83.730 & 0.8844 & 33.826 & 0.4549 & 17.036 & 0.2892 & 12.8711 & 0.1809 \\
\hline
\end{tabular}

TABLE II. 【VAR OLS (B^ $\left.\ \_0\right)$ WHEN $\Sigma=1$

\begin{tabular}{|c|c|c|c|c|}
\hline \multirow{2}{*}{$\begin{array}{c}\text { Sample } \\
\text { Size }\end{array}$} & \multicolumn{4}{|c|}{ Rho } \\
\cline { 2 - 5 } & $\mathbf{- 0 . 9 0}$ & $\mathbf{- 0 . 5 0}$ & $\mathbf{0 . 5 0}$ & $\mathbf{0 . 9 0}$ \\
\hline 50 & 0.1169 & 0.0283 & 0.2493 & 112.3288 \\
\hline 100 & 0.0539 & 0.0137 & 0.1118 & 26.4752 \\
\hline 200 & 0.0246 & 0.0063 & 0.0521 & 9.4950 \\
\hline 300 & 0.0174 & 0.0044 & 0.0328 & 5.5008 \\
\hline 500 & 0.0105 & 0.0022 & 0.0187 & 6.2541 \\
\hline
\end{tabular}

Fig. 1. 『Var OLS $\left(\beta^{\wedge} \rrbracket \_0\right)$ when $\sigma=1$

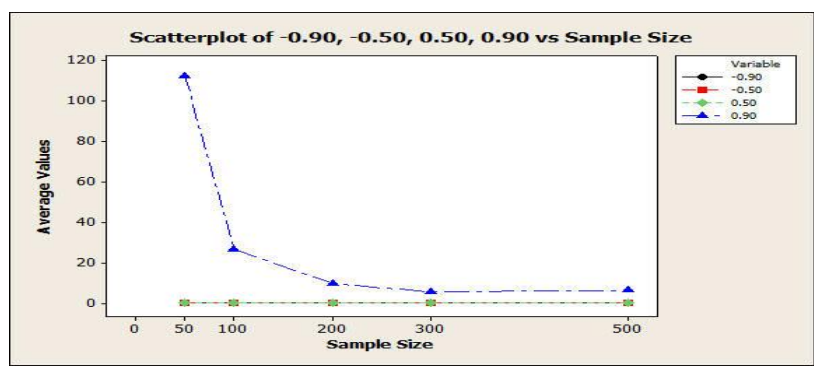

In Fig. 1 the variance of OLS ( $\left.\beta \_0\right)$, we observe that in different sample sizes $(50,100,200,300,500)$ and $\sigma=1$ with different correlation levels $(-0.90,-0.50,0.50,0.90)$. In sample size 500 with correlation level -0.50 the variance of OLS $\left(\beta_{-} 0\right)$ is 0.0022 , which is less than all others, and in sample size 50 with $\sigma=1$ the correlation level 0.90 have maximum value of variance OLS $\left(\beta \_0\right)$ is 112.3288 .

TABLE III. 『VAR OLS (B^^_1) WHEN $\Sigma=1$

\begin{tabular}{|c|c|c|c|c|}
\hline \multirow{2}{*}{$\begin{array}{c}\text { Sample } \\
\text { Size }\end{array}$} & \multicolumn{4}{|c|}{ Rho } \\
\cline { 2 - 5 } & $\mathbf{- 0 . 9 0}$ & $\mathbf{- 0 . 5 0}$ & $\mathbf{0 . 5 0}$ & $\mathbf{0 . 9 0}$ \\
\hline 50 & 0.3604 & 0.0785 & 0.2438 & 5.2785 \\
\hline 100 & 0.1704 & 0.0438 & 0.1070 & 3.2579 \\
\hline 200 & 0.0710 & 0.0186 & 0.0466 & 1.5402 \\
\hline 300 & 0.0497 & 0.0497 & 0.0264 & 0.8131 \\
\hline 500 & 0.0316 & 0.0316 & 0.0171 & 0.5811 \\
\hline
\end{tabular}

Fig. 2. $\llbracket \operatorname{Var} \mathrm{OLS}\left(\beta^{\wedge} \rrbracket \_1\right)$ when $\sigma=1$

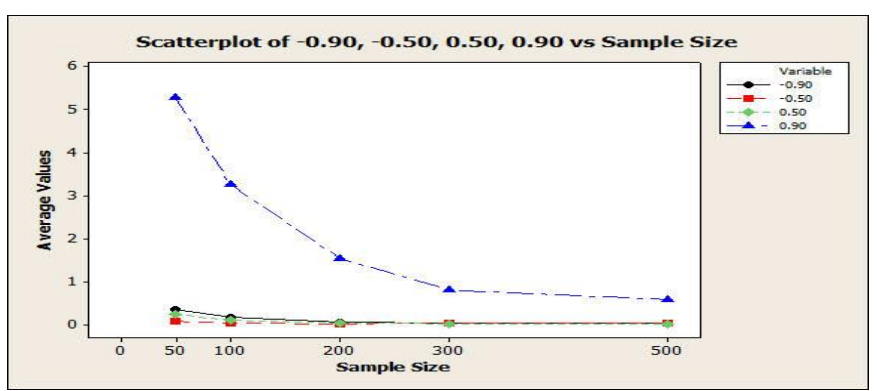

In Fig. 2 the variance of OLS $\left(\beta_{-} 1\right)$, we observe that in different sample sizes $(50,100,200,300,500)$ and $\sigma=1$ with different correlation levels $(-0.90,-0.50,0.50,0.90)$. In sample size 500 with correlation level 0.50 the variance of OLS is 0.0171 , which is less than all others, and in sample size 50 with $\sigma=1$ the correlation level 0.90 have maximum value of variance OLS $\left(\beta \_1\right)$ is 5.2785 .

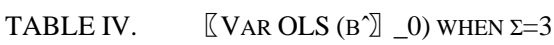

\begin{tabular}{|c|c|c|c|c|}
\hline \multirow{2}{*}{$\begin{array}{c}\text { Sample } \\
\text { Size }\end{array}$} & \multicolumn{4}{|c|}{ Rho } \\
\cline { 2 - 5 } & $\mathbf{- 0 . 9 0}$ & $\mathbf{- 0 . 5 0}$ & $\mathbf{0 . 5 0}$ & $\mathbf{0 . 9 0}$ \\
\hline 50 & 0.3293 & 0.0841 & 0.7669 & 282.6318 \\
\hline 100 & 0.1589 & 0.0412 & 0.3286 & 92.0552 \\
\hline 200 & 0.0717 & 0.0191 & 0.1479 & 35.5994 \\
\hline 300 & 0.0516 & 0.0127 & 0.0970 & 17.5397 \\
\hline 500 & 0.0295 & 0.0063 & 0.0601 & 13.1078 \\
\hline
\end{tabular}

Fig. 3. $\llbracket \operatorname{Var} \operatorname{OLS}\left(\beta^{\wedge} \rrbracket \_0\right)$ when $\sigma=3$

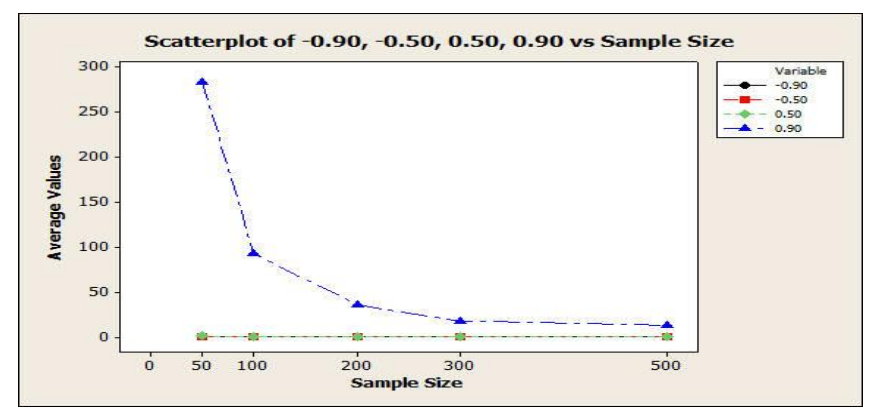

In Fig. 3 the variance of OLS $\left(\beta_{0}\right)$, we observe that in different sample sizes $(50,100,200,300,500)$ and $\sigma=3$ with different correlation levels $(-0.90,-0.50,0.50,0.90)$. In sample size 500 with correlation level -0.50 the variance of $\operatorname{OLS}\left(\beta_{0}\right)$ is 0.0063 , which is less than all others, and in sample size 50 with $\sigma=3$ the correlation level 0.90 have maximum value of variance OLS $\left(\beta_{0}\right)$ is 282.6318 .

TABLE V. 【VAR OLS (B`^_1) WHEN $\Sigma=3$

\begin{tabular}{|c|c|c|c|c|}
\hline \multirow{2}{*}{$\begin{array}{c}\text { Sample } \\
\text { Size }\end{array}$} & $\mathbf{- 0 . 9 0}$ & $\mathbf{- 0 . 5 0}$ & $\mathbf{0 . 5 0}$ & $\mathbf{0 . 9 0}$ \\
\cline { 2 - 5 } & & & & \\
\hline 50 & 1.1039 & 0.2532 & 0.4782 & 19.2061 \\
\hline 100 & 0.4966 & 0.1140 & 0.2786 & 9.9035 \\
\hline 200 & 0.1998 & 0.0509 & 0.1668 & 3.9800 \\
\hline 300 & 0.0497 & 0.0383 & 0.0902 & 2.3885 \\
\hline 500 & 0.0839 & 0.0198 & 0.0572 & 1.6248 \\
\hline
\end{tabular}

Fig. 4. $\square \operatorname{Var}$ OLS $(\beta \rrbracket]$ _) when $\sigma=3$

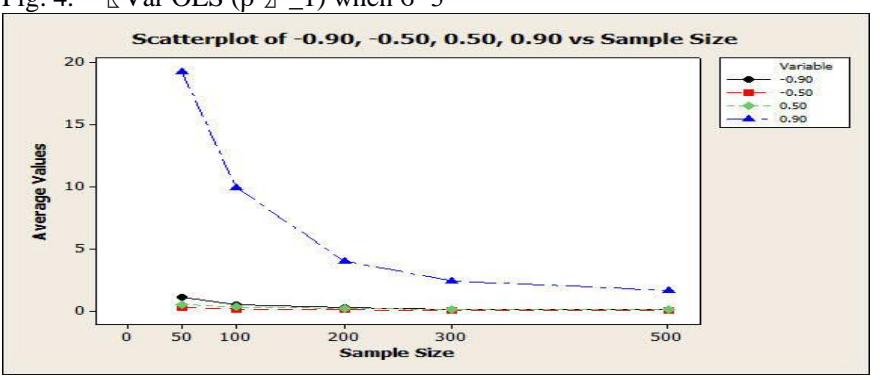


In Fig. 4 the variance of OLS ( $\beta$ _ 1$)$, we observe that in different sample sizes $(50,100,200,300,500)$ and $\sigma=3$ with different correlation levels $(-0.90,-0.50,0.50,0.90)$. In sample size 500 with correlation level -0.50 the variance of OLS is 0.0198 , which is less than all others, and in sample size 50 with $\sigma=3$ the correlation level 0.90 have maximum value of variance OLS ( $\left.\beta \_1\right)$ is 19.2061.

TABLE VI. 『VAR GLS (B^^_0) WHEN $\Sigma=1$

\begin{tabular}{|c|c|c|c|c|}
\hline \multirow{2}{*}{$\begin{array}{c}\text { Sample } \\
\text { Size }\end{array}$} & \multicolumn{4}{|c|}{ Rho } \\
\cline { 2 - 5 } & $\mathbf{- 0 . 9 0}$ & $\mathbf{- 0 . 5 0}$ & $\mathbf{0 . 5 0}$ & $\mathbf{0 . 9 0}$ \\
\hline 50 & 0.1168 & 0.0283 & 0.2080 & 105.4205 \\
\hline 100 & 0.0539 & 0.0137 & 0.1108 & 24.1893 \\
\hline 200 & 0.0246 & 0.0063 & 0.0521 & 9.0764 \\
\hline 300 & 0.0174 & 0.0044 & 0.0328 & 5.3486 \\
\hline 500 & 0.0105 & 0.0022 & 0.0186 & 6.1088 \\
\hline
\end{tabular}

Fig. 5. 『Var GLS $\left(\beta^{\wedge} \rrbracket \_0\right)$ when $\sigma=1$

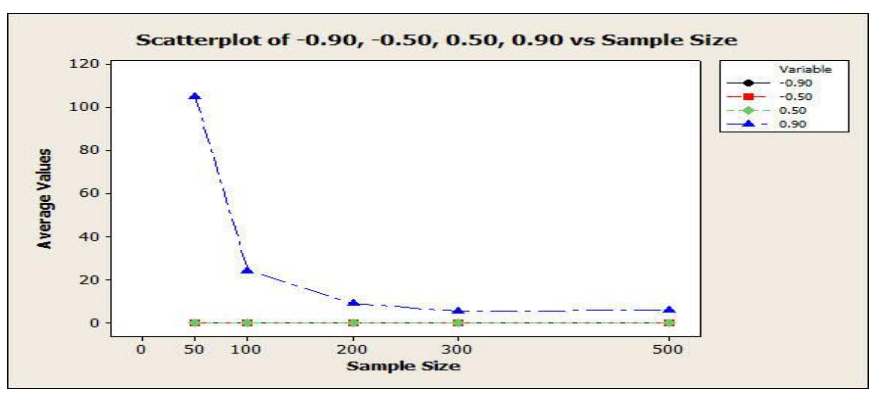

In Fig. 5 the variance of GLS $(\beta 0)$, we observe that in different sample sizes $(50,100,200,300,500)$ and $\sigma=1$ with different correlation levels $(-0.90,-0.50,0.50,0.90)$. In sample size 500 with correlation level -0.50 the variance of GLS $\left(\beta \_0\right)$ is 0.0022 , which is less than all others, and in sample size 50 with $\sigma=1$ the correlation level 0.90 have maximum value of variance GLS $\left(\beta \_0\right)$ is 105.4205 .

TABLE VII. 『VAR GLS (B^^_1) WHEN $\Sigma=1$

\begin{tabular}{|c|c|c|c|c|}
\hline \multirow{2}{*}{$\begin{array}{c}\text { Sample } \\
\text { Size }\end{array}$} & $\mathbf{- 0 . 9 0}$ & $\mathbf{- 0 . 5 0}$ & $\mathbf{0 . 5 0}$ & $\mathbf{0 . 9 0}$ \\
\cline { 2 - 5 } & & & & 0.1197 \\
\hline 50 & 0.3604 & 0.0785 & 0.5324 \\
\hline 100 & 0.1704 & 0.0438 & 0.0605 & 0.3490 \\
\hline 200 & 0.0710 & 0.0186 & 0.0265 & 0.1366 \\
\hline 300 & 0.0497 & 0.0133 & 0.0168 & 0.0853 \\
\hline 500 & 0.0316 & 0.0064 & 0.0099 & 0.0587 \\
\hline
\end{tabular}

Fig. 6. \Var GLS $\left(\beta^{\wedge} \rrbracket \_1\right)$ when $\sigma=1$

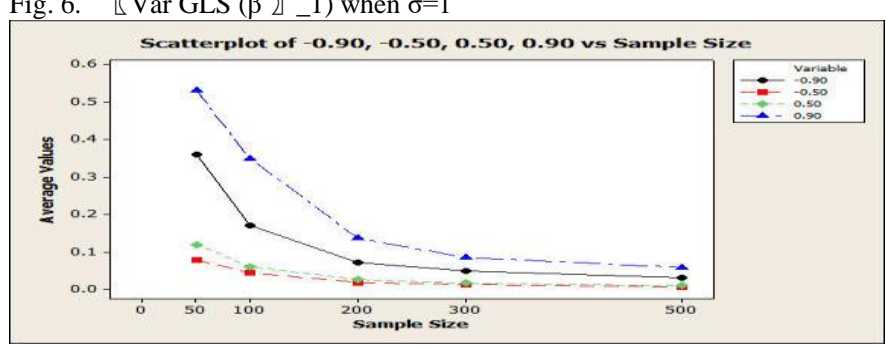

In Fig. 6 the variance of GLS $\left(\beta_{-} 1\right)$, we observe that in different sample sizes $(50,100,200,300,500)$ and $\sigma=1$ with different correlation levels $(-0.90,-0.50,0.50,0.90)$. In sample size 500 with correlation level -0.50 the variance of
GLS ( $\left.\beta \_1\right)$ is 0.0064 , which is less than all others, and in sample size 50 with $\sigma=1$ the correlation level 0.90 have maximum value of variance GLS $\left(\beta_{-} 1\right)$ is 0.5324 .

TABLE VIII. 『VAR GLS (B^^_0) WHEN $\Sigma=3$

\begin{tabular}{|c|c|c|c|c|}
\hline \multirow{2}{*}{$\begin{array}{c}\text { Sample } \\
\text { Size }\end{array}$} & \multicolumn{4}{|c|}{ Rho } \\
\cline { 2 - 5 } & $\mathbf{- 0 . 9 0}$ & $\mathbf{- 0 . 5 0}$ & $\mathbf{0 . 5 0}$ & $\mathbf{0 . 9 0}$ \\
\hline 50 & 0.3293 & 0.0841 & 0.7513 & 261.4951 \\
\hline 100 & 0.1589 & 0.0412 & 0.3249 & 83.7309 \\
\hline 200 & 0.0717 & 0.0191 & 0.1469 & 33.8265 \\
\hline 300 & 0.0516 & 0.0127 & 0.0968 & 17.0360 \\
\hline 500 & 0.0295 & 0.0063 & 0.0600 & 12.8711 \\
\hline
\end{tabular}

Fig. 7. 『Var GLS $\left(\beta^{\wedge} \rrbracket \_0\right)$ when $\sigma=3$

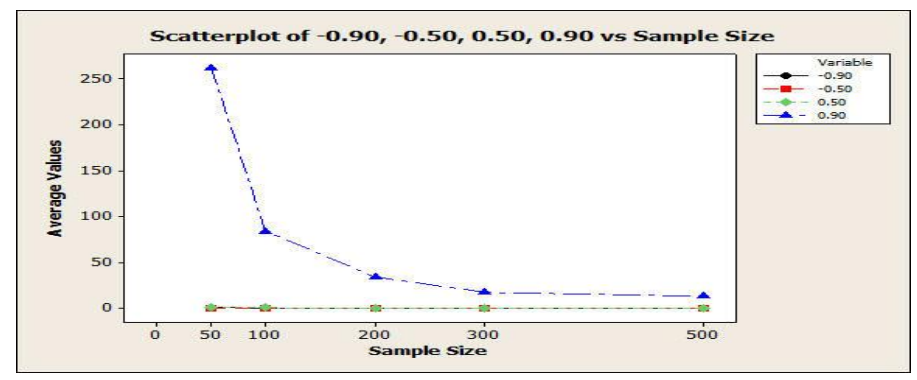

In Fig. 7 the variance of GLS $\left(\beta \_0\right)$, we observe that in different sample sizes $(50,100,200,300,500)$ and $\sigma=3$ with different correlation levels $(-0.90,-0.50,0.50,0.90)$. In sample size 500 with correlation level -0.50 the variance of GLS $\left(\beta \_0\right)$ is 0.0063 , which is less than all others, and in sample size 50 with $\sigma=3$ the correlation level 0.90 have maximum value of variance GLS $\left(\beta \_0\right)$ is 261.4951 .

TABLE IX. 『VAR GLS (B^^_1) WHEN $\Sigma=3$

\begin{tabular}{|c|c|c|c|c|}
\hline \multirow{2}{*}{$\begin{array}{c}\text { Sample } \\
\text { Size }\end{array}$} & \multicolumn{4}{|c|}{ Rho } \\
\cline { 2 - 5 } & $\mathbf{- 0 . 9 0}$ & $\mathbf{- 0 . 5 0}$ & $\mathbf{0 . 5 0}$ & $\mathbf{0 . 9 0}$ \\
\hline 50 & 1.1038 & 0.2532 & 0.2837 & 2.1179 \\
\hline 100 & 0.4965 & 0.1140 & 0.1572 & 0.8844 \\
\hline 200 & 0.1997 & 0.0509 & 0.0921 & 0.4549 \\
\hline 300 & 0.1533 & 0.0383 & 0.0547 & 0.2892 \\
\hline 500 & 0.8394 & 0.0198 & 0.0320 & 0.1809 \\
\hline
\end{tabular}

Fig. 8. 『Var GLS $\left(\beta^{\wedge} \rrbracket \_1\right)$ when $\sigma=3$

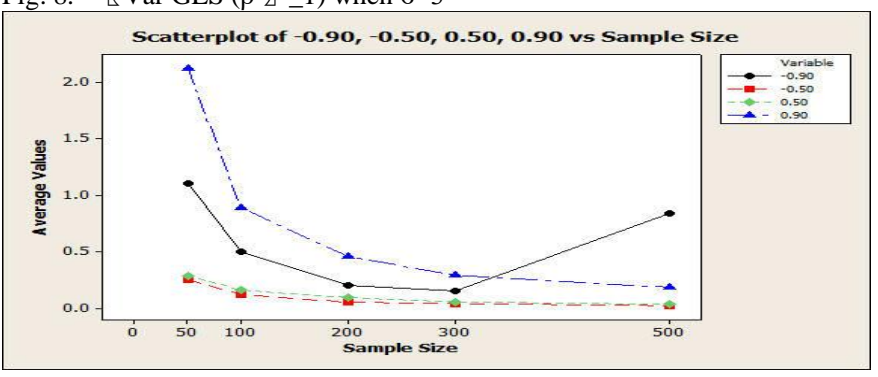

In Fig. 8 the variance of GLS $(\beta 1)$, we observe that in different sample sizes $(50,100,200,300,500)$ and $\sigma=3$ with different correlation levels $(-0.90,-0.50,0.50,0.90)$. In sample size 500 with correlation level -0.50 the variance of GLS ( $\left.\beta \_1\right)$ is 0.0198 , which is less than all others, and in sample size 50 with $\sigma=3$ the correlation level 0.90 have maximum value of variance GLS $\left(\beta \_1\right)$ is 2.1179 . 


\section{CONCLUSION}

The average values of parameters of the Ordinary Least Squares and Generalized Least Squares estimation with different size of sample and correlation levels are estimated. When the bias values of Ordinary Least Squares and Generalized Least Squares is not normal with haphazard manner of average values.

By comparing variances of OLS and GLS at different values of sample sizes and correlation levels with $\sigma=1,3$, we found that variance of $\left(\beta \_0\right)$ at sample size 500, OLS and GLS gives similar results but at sample size 50 variance of GLS ( $\left.\beta \_0\right)$ has minimum values as compared to OLS. So it is clear that variance of GLS $\left(\beta \_0\right)$ is best. Similarly variance of $(\beta 1)$ from OLS and GLS at sample size 500 and correlation -0.05 with $\sigma=1$, GLS give minimum value as compared to all other sample sizes and correlations.

By comparing overall results of Ordinary Least Squares (OLS) and Generalized Least Squares (GLS), we conclude that in large samples both are gives similar results but small samples GLS is best fitted as compared to OLS.

\section{REFERENCES}

[1] Aitken, A. C. (1935). On Least Squares and Linear Combinations of Observations. Proceedings of the Royal Statistical Society , 55, 42-48.

[2] Akpan, E. A., \& Moffat, I. U. (2018). Modeling the Autocorrelated Errors in Time Series Regression: A Generalized Least Squares
Approach. Journal of Advances in Mathematics and Computer Science, 26 (4), 1-15

[3] Chang, X. W., \& Peloquin, D. T. (2020). An Improved Algorithm for Generalized Least Squares Estimation. Numerical Algebra, Control and Optimization , 10 (4), 451-461.

[4] Kassab, M. M., \& Awjar, M. Q. (2020). A Monte Carlo Comparison between Least Squares and the New Ridge Regression Parameters. Advances and Applications in Statistics , 62 (1), 97-105.

[5] Kibria, B. M., \& Lukman, A. F. (2020). A New Ridge-Type Estimator for the Linear Regression Model: Simulations and Applications. Scientifica, 1-16.

[6] Liu, K. (1993). A New Class of Biased Estimate in Linear Regression. Communication in Statistics- Theory and Methods , 22, 393-402.

[7] Moon, T. K., \& Gunther, J. H. (2020). Estimation of Autoregressive Parameters from Noisy Observations Using Iterated Covariance Updates. Entropy , 1-26.

[8] Safi, S. K., \& Saif, E. A. (2014). Using GLS to Generate Forecasts in Regression Models with Auto-Correlated Disturbances with Simulation and Palestinian Market Index Data. American Journal of Theoretical and Applied Statistics , 3 (1), 6-17.

[9] Safi, S., \& White, A. (2006). The Efficiency of OLS in the Presence of Auto-Correlated Disturbances in Regression Models. Journal of Modern Applied Statistical Methods , 5 (1), 133-143.

[10] Virgantari, F., Wijayanti, H., \& Koeshendrajana, S. (2018). Aitken's Generalized Least Square Method for Estimating Parameter of Demand Function of Animal Protein in Indonesia. International Conference on Mathematics and Natural Sciences (pp. 1-8). Journal of Physics.

[11] Wenning, Z., \& Valenci, E. (2019). A Monte Carlo Simulation Study on the Power of Autocorrelation Tests for ARMA Models. American Journal of Undergraduate Research , 16 (3), 59-67. 